\title{
A quadratic point on the Jacobian of the universal genus four curve
}

\author{
HANG XUE
}

In this paper, we construct a point on the Jacobian of a nonhyperelliptic genus four curve which is defined over a quadratic extension of the base field. We then show that this point generates the Mordell-Weil group of the Jacobian of the universal genus four curve.

\section{Statement of the theorem}

Let $K$ be a field such that char $K \neq 2$. Let $X$ be a non-hyperelliptic curve of genus four over $K$. Then $X$ is a complete intersection of $Q$ and $S$ in $\mathbb{P}^{3}$, where $Q$ is a (unique) geometrically irreducible quadric surface and $S$ is a geometrically irreducible cubic surface [6, Chapter IV, Example 5.5.2]. In fact, if $Q$ is reducible, then it is a union of two planes in $\mathbb{P}^{3}$ and this implies that $X$ lies in a plane. This is a contradiction since $X$ is non-hyperelliptic. Similarly we can show that $S$ is geometrically irreducible.

Assume that $Q$ is smooth. Let $\bar{K}$ be the algebraic closure of $K$. It is well-known that $Q$ is isomorphic to $\mathbb{P}^{1} \times \mathbb{P}^{1}$ over $\bar{K}$. Let $j: X \rightarrow Q$ be the embedding. Then $\Xi=j^{*} \mathcal{O}(1,-1)$ is a degree zero line bundle on $X$ which gives an element in $\operatorname{Jac}(X)(\bar{K})$. Note that $\Xi$ is defined up to a sign, since one can switch the two $\mathbb{P}^{1}$ 's in the isomorphism $Q \simeq \mathbb{P}^{1} \times \mathbb{P}^{1}$ over $\bar{K}$. When $Q$ is not smooth, we define $\Xi \in \operatorname{Jac}(X)(\bar{K})$ to be zero.

Remark 1.1. Assume that $Q$ is smooth. Over $\bar{K}$, there are two degree three one dimensional linear systems on $X$. They are cut out by the two rulings of $Q$ respectively. Then $\Xi$ is the difference between the divisors in these two linear systems.

Lemma 1.2. Let $\operatorname{disc} Q$ be the discriminant of $Q$ and $K^{\prime}=K(\sqrt{\operatorname{disc} Q})$. Then the point $\Xi$ is defined over $K^{\prime}$.

Proof. We may assume that $Q$ is smooth. The case $Q$ being singular is automatic since $\Xi$ is trivial. If $\operatorname{disc} Q \in K^{\times, 2}$, then we claim that $Q$ admits a 
fibration $Q \rightarrow C$ where the fibers are all $\mathbb{P}^{1}$ 's and $C$ is a genus zero curve over $K$. In fact, without loss of generality, we may assume that $Q$ is defined by the equation $x^{2}+a y^{2}+b z^{2}+a b w^{2}=0(\operatorname{char} K \neq 2)$. If $-a b \in K^{\times, 2}$, then by a suitable change of variables, we see that $Q$ is isomorphic to $\mathbb{P}^{1} \times \mathbb{P}^{1}$ over $K$. If $-a b \notin K^{\times, 2}$, then we let $L=K(\sqrt{-a b})$. There is morphism

$$
Q \rightarrow \operatorname{Res}_{L / K} \mathbb{P}^{1}, \quad(x, y, z, w) \mapsto\left(x+\sqrt{-a b} w, y+a^{-1} \sqrt{-a b} z\right),
$$

whose image is a curve in $\operatorname{Res}_{L / K} \mathbb{P}^{1}$ defined by the equation $X X^{\tau}+a Y Y^{\tau}=$ 0 where $\tau$ is the nontrivial element in $\operatorname{Gal}(L / K)$. This curve is of genus zero and is the desired curve $C$ in the claim.

We then get a morphism $j: X \rightarrow C$. Let $\omega_{C}$ be the dualizing sheaf of $C$ over Spec $K$. Then $\omega_{X} \otimes j^{*} \omega_{C}$ is a line bundle on $X$ defined over $K$ and it coincides with $\Xi$ over $\bar{K}$. In this case, we have $K^{\prime}=K$ and the point $\Xi$ is defined over $K$.

If $\operatorname{disc} Q \notin K^{\times, 2}$, then let $K^{\prime}=K(\sqrt{\operatorname{disc} Q})$. The point $\Xi$ is then defined over $K^{\prime}$ by the argument in the previous paragraph.

Our main theorem of this paper is the following.

Theorem 1.3. Let $k$ be a field of characteristic not two or three and let $\mathcal{M}_{4}$ be the moduli space of genus four curves over $k$. Let $K$ be the function field of $\mathcal{M}_{4}$. Let $\mathcal{C}_{4} \rightarrow \mathcal{M}_{4}$ be the universal curve and let $C_{4} \rightarrow \operatorname{Spec} K$ be its generic fiber. Then $\operatorname{Jac}\left(C_{4}\right)\left(K^{\prime}\right) \simeq \mathbb{Z}$ and is generated by $\Xi$.

Remark 1.4. This theorem is related to the Franchetta's conjecture. This conjecture claims that $\operatorname{Jac}\left(C_{4}\right)(K)=0$ in our case. Since $\Xi^{\tau}=-\Xi$ where $\tau$ is the nonzero element in $\operatorname{Gal}\left(K^{\prime} / K\right)$, any multiple of $\Xi$ does not descend to a point in $\operatorname{Jac}\left(C_{4}\right)(K)$. Thus we have reproved Franchetta's conjecture for the universal genus four curve. We point out that the method in this paper does not involve degeneration argument and does not depend on a priori information of the Picard group of any moduli space. This is different from the techniques in the existing proofs of the Franchetta's conjecture.

Remark 1.5. We are not sure if the hypothesis char $k \neq 3$ is necessary. This hypothesis is only used in the Step 2 of the Proof of Proposition 2.2. In the case of char $k=3$, we get the slightly weaker statement that $\operatorname{Jac}\left(C_{4}\right)\left(K^{\prime}\right) \simeq$ $\mathbb{Z} \oplus T$ where $T=0$ or $T=\mathbb{Z} / 3 \mathbb{Z}$ and $\Xi$ generates $\operatorname{Jac}\left(C_{4}\right)\left(K^{\prime}\right) / T$.

Notation. Let $F$ be a field, then we denote by $\bar{F}$ its algebraically closure. If $X \rightarrow Y$ is a morphism of schemes and $L$ is a line bundle on $Y$, then we 
denote by $\left.L\right|_{X}$ the pullback of $L$ to $X$. If $X \rightarrow S$ and $T \rightarrow S$ are $S$-schemes, then we denote by $X_{T}=X \times_{S} T$ the base change of $X$ to $T$.

\section{Proof of Theorem 1.3}

We fix a base field $k$ of characteristic $p$ with $p=0$ or $p \geq 5$. Without loss of generality, we may assume that $k$ is algebraically closed. By a curve of type $(m, n)$ in $\mathbb{P}^{1} \times \mathbb{P}^{1}$, or an $(m, n)$ curve, we mean the curve defined by a bihomogeneous equation of degree $(m, n)$ in $\mathbb{P}^{1} \times \mathbb{P}^{1}$. Let $P(m, n)$ be the space of $(m, n)$ curves in $\mathbb{P}^{1} \times \mathbb{P}^{1}$.

The moduli space $\mathcal{M}_{4}$ contains an open subscheme $\mathcal{M}_{4}^{\text {ns }}$ parameterizing smooth genus four curves whose canonical images are smooth $(3,3)$ curves in $\mathbb{P}^{1} \times \mathbb{P}^{1}$. A $(3,3)$ curve is given by an equation

$$
\sum_{0 \leq i, j \leq 3} a_{i j} x_{0}^{i} x_{1}^{3-i} y_{0}^{j} y_{1}^{3-j}=0 .
$$

Then $P(3,3)$ is isomorphic to the projective space of the coefficients $\mathbb{P}\left[a_{i j}\right.$ : $0 \leq i, j \leq 3]$. The group $\mathrm{PGL}_{2} \times \mathrm{PGL}_{2} \rtimes\langle \pm 1\rangle$ acts on $P(3,3)$, where the two $\mathrm{PGL}_{2}$ factors act by changing coordinates on the two $\mathbb{P}^{1}$ 's respectively and $\langle \pm 1\rangle$ acts by sending $a_{i j}$ to $a_{j i}$. Let $P(3,3)^{0}$ be the open subscheme of $P(3,3)$ parameterizing smooth $(3,3)$-curves in $\mathbb{P}^{1} \times \mathbb{P}^{1}$. Then $\mathcal{M}_{4}^{\text {ns }}$ is the quotient of $P(3,3)^{0}$ by $\mathrm{PGL}_{2} \times \mathrm{PGL}_{2} \rtimes\langle \pm 1\rangle$. Let $\widetilde{\mathcal{M}_{4}^{\text {ns }}}$ be the quotient of $P(3,3)^{0}$ by $\mathrm{PGL}_{2} \times \mathrm{PGL}_{2}$. Then $K^{\prime}$ is the function field of $\widetilde{\mathcal{M}_{4}^{\text {ns }}}$.

Let $Y=\mathbb{P}^{1} \times \mathbb{P}^{1} \times \mathbb{P}^{1}$ and let $\mathcal{L}=\mathcal{O}(d, 3,3)$ be a line bundle on $Y$. Assume that $d \geq 2$ and is prime to $p$. The line bundle $\mathcal{L}$ is very ample and induces an embedding $Y \rightarrow \mathbb{P}^{N}$. The linear system $|\mathcal{L}|$ parameterizes all hyperplane sections of $Y$. A hyperplane section $X$ of $Y$ admits a morphism $X \rightarrow \mathbb{P}^{1}$ via the projection to the first factor $\mathbb{P}^{1}$ of $Y$. Let $U \subset|\mathcal{L}|$ be the open subset parameterizing smooth hyperplane sections of $Y$ which are flat over $\mathbb{P}^{1}$.

Lemma 2.1. There is a non-empty open subset $V \subset U$, such that if a hyperplane section lies in $V$, then as a fibration over $\mathbb{P}^{1}$, it is not isotrivial and all the fibers are irreducible.

Proof. Any hyperplane section $X$ in $U$ gives rise to a morphism $\mathbb{P}^{1} \rightarrow$ $P(3,3)$. Thus there is a dominant morphism $U \times \mathbb{P}^{1} \rightarrow P(3,3)$. The fibration $X \rightarrow \mathbb{P}^{1}$ is isotrivial if and only if the image of $\mathbb{P}^{1} \rightarrow P(3,3)$ lies in the closure of an orbit of $\mathrm{PGL}_{2} \times \mathrm{PGL}_{2} \rtimes\langle \pm 1\rangle$. Such hyperplane sections form a closed subset of $U$. It is clear that there is a hyperplane section $X$ which 
is not isotrivial as a fibration over $\mathbb{P}^{1}$. Let $U_{0}$ be the nonempty open subset of $U$ parameterizing hyperplane sections $X$ such that the fibration $X \rightarrow \mathbb{P}^{1}$ is not isotrivial.

The reducible $(3,3)$ curves form a proper algebraic subset $R$ of $P(3,3)$, namely the union of $P(a, b) \times P(3-a, 3-b)$, where $0 \leq a \leq 3,0 \leq b \leq 3, a$ and $b$ are not both 0 or 3 . Let $Z$ be the inverse image of $R$ in $U_{0} \times \mathbb{P}^{1}$. This is a proper algebraic subset of $U_{0} \times \mathbb{P}^{1}$ such that $(X, x) \in Z$ if and only if the fiber of $X \rightarrow \mathbb{P}^{1}$ over $x \in \mathbb{P}^{1}$ is reducible. Let $W$ be the image of $Z$ in $U_{0}$. Since $\mathbb{P}^{1}$ is proper, $W$ is a closed subset of $U_{0}$. We claim that $W \neq U_{0}$. Let $X^{\prime} \rightarrow \ell=\mathbb{P}^{1}$ be a Lefschetz pencil of $(3,3)$ curves in $\mathbb{P}^{1} \times \mathbb{P}^{1}$. The fiber of $X^{\prime} \rightarrow \ell$ are all irreducible. In fact, a singular fiber of $X^{\prime} \rightarrow \ell$ has most two components. If it had two components, then there would be at least $a(3-b)+b(3-a) \geq 3$ singular points on the fiber, which contradicts the definition of a Lefshetz pencil. Let $p_{d}: \mathbb{P}^{1} \rightarrow \mathbb{P}^{1}$ be a degree $d$ flat morphism which is etale over each point of the target $\mathbb{P}^{1}$ over which our Lefshcetz pencil has a singular fiber. Then the fiber product of $X^{\prime} \rightarrow \ell$ and $p_{d}: \mathbb{P}^{1} \rightarrow \mathbb{P}^{1}$ is an element in $U_{0}$ but not in $W$.

Thus $V=U_{0} \backslash W$ is a non-empty open subset of $U$ such that if $X$ lies in $V$, then $X \rightarrow \mathbb{P}^{1}$ is not isotrivial and all the fibers of $X \rightarrow \mathbb{P}^{1}$ are irreducible.

Let $\mathbb{X} \rightarrow V$ be the universal hyperplane section of $Y$ over $V$. Let $\mathcal{K}$ be the function field of $V$. Let $\eta=\operatorname{Spec} \mathcal{K}$ (resp. $\bar{\eta}=\operatorname{Spec} \overline{\mathcal{K}}$ ) be the generic (resp. geometric generic) point of $V$. Let $X_{\bar{\eta}} \rightarrow$ Spec $\overline{\mathcal{K}}$ be the geometric generic fiber of $\mathbb{X} \rightarrow V$. Let $f: X_{\bar{\eta}} \rightarrow \mathbb{P} \frac{1}{\bar{\eta}}$ be the projection to the first factor of $Y_{\bar{\eta}}$.

Let $\operatorname{NS}\left(X_{\bar{\eta}}\right)$ be the Neron-Severi group of $X_{\bar{\eta}}$. Let $\operatorname{NS}\left(X_{\bar{\eta}}\right)_{0}$ be the subgroup of $\mathrm{NS}\left(X_{\bar{\eta}}\right)$ consists of line bundles that are of degree zero on the generic fiber of $f$. Let $F$ be a smooth fiber of $f$ and $\Xi_{\bar{\eta}}=\left.\mathcal{O}(0,1,-1)\right|_{X_{\bar{\eta}}}$.

Proposition 2.2. The group $\mathrm{NS}\left(X_{\bar{\eta}}\right)_{0}$ is a free abelian group of rank two and is generated by $F$ and $\Xi_{\bar{\eta}}$.

Proposition 2.2 will be proved in the next section.

Let $L$ be the function field of $\mathbb{P}_{\bar{\eta}}$. Let $X_{L}$ be the generic fiber of $f$. Then $\Xi_{\bar{\eta}}$ gives an element in $\operatorname{Jac}\left(X_{L}\right)(L)$ which we denote by $\Xi_{L}$.

Proposition 2.3. The group $\operatorname{Jac}\left(X_{L}\right)(L)$ is isomorphic to $\mathbb{Z}$ and is generated by $\Xi_{L}$.

Proof. We first note that $L$ is the function field of a curve over an algebraically closed constant field. Thus the Brauer group $\operatorname{Br}(L)=0$ (Tsen's Theorem) and the elements in $\operatorname{Jac}\left(X_{L}\right)(L)$ are represented by line bundles 
on $X_{L}$ [4, Section 8.1]. Therefore the map $\operatorname{NS}\left(X_{\bar{\eta}}\right)_{0} \rightarrow \operatorname{Jac}\left(X_{L}\right)(L)$ is surjective. As an element in $\operatorname{NS}\left(X_{\bar{\eta}}\right)$, the self-intersection of $\Xi_{\bar{\eta}}$ is $-2 d \neq 0$. Since the fibers of $f$ are all irreducible, $\Xi_{L}$ is not torsion in $\operatorname{Jac}\left(X_{L}\right)(L)$. Therefore the kernel of the map $\operatorname{NS}\left(X_{\bar{\eta}}\right)_{0} \rightarrow \operatorname{Jac}\left(X_{L}\right)(L)$ is generated by $F$. It then follows that $\operatorname{Jac}\left(X_{L}\right)(L)$ is isomorphic to $\mathbb{Z}$ and is generated by $\Xi_{L}$.

Proof of Theorem 1.3. The canonical morphism $\mathbb{P}^{1} \times V \rightarrow P(3,3)$ is dominant. Therefore $L$ is a (large transcendental) field extension of $K^{\prime}$. If follows that $\operatorname{Jac}\left(X_{L}\right) \simeq \operatorname{Jac}\left(C_{4}\right) \otimes_{K^{\prime}} L$ and $\operatorname{Jac}\left(C_{4}\right)\left(K^{\prime}\right)$ injects into $\operatorname{Jac}\left(X_{L}\right)(L)$. The image of $\Xi$ in $\operatorname{Jac}\left(X_{L}\right)(L)$ is $\Xi_{L}$. Theorem 1.3 then follows from Proposition 2.3 .

\section{Proof of Proposition 2.2}

Let $l \neq p$ be a prime. The proof of Proposition 2.2 is divided into two steps.

Step 1: The restriction map $\mathrm{NS}\left(Y_{\bar{\eta}}\right) \otimes \mathbb{Q} \rightarrow \mathrm{NS}\left(X_{\bar{\eta}}\right) \otimes \mathbb{Q}$ is an isomorphism.

Proof. The following argument is similar to [1, Exposé XIX]. The restriction map $\mathrm{H}^{2}\left(Y_{\bar{\eta}}, \mathbb{Q}_{l}(1)\right) \rightarrow \mathrm{H}^{2}\left(X_{\bar{\eta}}, \mathbb{Q}_{l}(1)\right)$ is injective by the Weak Lefschetz Theorem. It is $\operatorname{Gal}(\overline{\mathcal{K}} / \mathcal{K})$-equivariant. The orthogonal complement of $\mathrm{H}^{2}\left(Y_{\bar{\eta}}, \mathbb{Q}_{l}(1)\right)$ in $\mathrm{H}^{2}\left(X_{\bar{\eta}}, \mathbb{Q}_{l}(1)\right)$ is denoted by E. There is a non-degenerate symmetric pairing on E. The image of $\operatorname{Gal}(\overline{\mathcal{K}} / \mathcal{K})$ in $\mathrm{GL}(\mathrm{E})$ lies in $\mathrm{O}(\mathrm{E})$. Moreover, the representation of $\mathrm{Gal}(\overline{\mathcal{K}} / \mathcal{K})$ on $\mathrm{E}$ is absolutely irreducible $[5$, $\S 5.5,5.8]$. We shall call the Zariski closure of the image of $\operatorname{Gal}(\overline{\mathcal{K}} / \mathcal{K})$ in $\mathrm{O}(\mathrm{E})$ the monodromy group.

We denote by $\mathrm{E}^{\text {alg }}$ the subspace of $\mathrm{E}$ generated by the cohomology classes of algebraic cycles on $X_{\bar{\eta}}$. This subspace is again preserved by the action of $\operatorname{Gal}(\mathcal{K} / \mathcal{K})$. Therefore either $E^{\text {alg }}=E$ or $E^{\text {alg }}=0$.

Lemma 3.1. If $\mathrm{E}=\mathrm{E}^{\mathrm{alg}}$, then the monodromy group is finite.

Proof. Every algebraic cycle on $X_{\bar{\eta}}$ is defined over some finite extension of $\mathcal{K}$. Therefore it is stabilized by an open subgroup of $\operatorname{Gal}(\overline{\mathcal{K}} / \mathcal{K})$ of finite index. Moreover the space $\mathrm{E}$ is finite dimensional. We then conclude that the action of $\operatorname{Gal}(\overline{\mathcal{K}} / \mathcal{K})$ factors through an open subgroup of finite index. Its image in $\mathrm{O}(\mathrm{E})$ is thus finite.

Lemma 3.2. If $p=0$, then $\mathrm{E}^{\mathrm{alg}}=0$ and the monodromy group is not finite.

Proof. Suppose $\mathrm{E}^{\mathrm{alg}} \neq 0$. Then $\mathrm{E}=\mathrm{E}^{\mathrm{alg}}$ and the monodromy group is finite by the previous lemma. We embed $\mathcal{K}$ into $\mathbb{C}$ and view $X_{\bar{\eta}}$ as a variety over $\mathbb{C}$. 
We fix an embedding $\mathbb{Q}_{l} \rightarrow \mathbb{C}$ and denote by $\mathrm{E}_{\mathbb{C}}$ the subspace of $\mathrm{H}^{2}\left(X_{\bar{\eta}}, \mathbb{C}\right)$ generated by $\mathrm{E}$. Then $\mathrm{E}_{\mathbb{C}} \subset \mathrm{H}^{1,1}\left(X_{\bar{\eta}}\right)$ since it is generated by the cohomology classes of algebraic cycles. So to get the contradiction, we only have to check that $\mathrm{H}^{2,0}\left(X_{\bar{\eta}}\right) \cap \mathrm{E}_{\mathbb{C}} \neq 0$.

Write $j: X_{\bar{\eta}} \rightarrow Y_{\bar{\eta}}$ for the embedding. By the adjunction formula, $\omega_{X_{\bar{\eta}}} \simeq$ $j^{*} \mathcal{O}(d-2,1,1)$. Then $\mathrm{H}^{2,0}=\mathrm{H}^{0}\left(Y_{\bar{\eta}}, \mathcal{O}(d-2,1,1) \otimes j_{*} \mathcal{O}_{X_{\bar{\eta}}}\right)$. By definition we have

$$
0 \rightarrow \mathcal{O}_{Y_{\bar{\eta}}}\left(-X_{\bar{\eta}}\right) \rightarrow \mathcal{O}_{Y_{\bar{\eta}}} \rightarrow j_{*} \mathcal{O}_{X_{\bar{\eta}}} \rightarrow 0,
$$

where $\mathcal{O}_{Y_{\bar{\eta}}}\left(-X_{\bar{\eta}}\right)=\mathcal{O}(-d,-3,-3)$. Therefore

$$
0 \rightarrow \mathcal{O}(-2,-2,-2) \rightarrow \mathcal{O}(d-2,1,1) \rightarrow \mathcal{O}(d-2,1,1) \otimes j_{*} \mathcal{O}_{X_{\bar{\eta}}} \rightarrow 0 .
$$

Taking long exact sequence gives

$$
0 \rightarrow \mathrm{H}^{0}\left(Y_{\bar{\eta}}, \mathcal{O}(d-2,1,1)\right) \rightarrow \mathrm{H}^{0}\left(Y_{\bar{\eta}}, \mathcal{O}(d-2,1,1) \otimes j_{*} \mathcal{O}_{X_{\bar{\eta}}}\right) .
$$

By the Künneth formula we get $\operatorname{dim} \mathrm{H}^{0}\left(Y_{\bar{\eta}}, \mathcal{O}(d-2,1,1)\right)=4 \operatorname{dim} \mathrm{H}^{0}\left(\mathbb{P}^{1}\right.$, $\mathcal{O}(d-2)) \geq 4$ since $d \geq 2$. This yields $\operatorname{dim} \mathrm{H}^{2,0}\left(X_{\bar{\eta}}\right) \geq 4$. Moreover, since $\operatorname{dim} \mathrm{H}^{2}\left(Y_{\bar{\eta}}, \mathbb{C}\right)=3$, we know that $\mathrm{E}_{\mathbb{C}}$ is of codimension 3 in $\mathrm{H}^{2}\left(X_{\bar{\eta}}, \mathbb{C}\right)$. Therefore $\mathrm{H}^{2,0}\left(X_{\bar{\eta}}\right) \cap \mathrm{E}_{\mathbb{C}} \neq 0$. This proves the lemma.

Lemma 3.3. If $p \geq 5$, then $\mathrm{E}^{\text {alg }}=0$.

Proof. We deduce the lemma from its characteristic zero counterpart. Let $R$ be a discrete valuation ring of mixed characteristic with the residue field $k$ and the fraction field $F$. The scheme $Y$ and the line bundle $\mathcal{L}$ are both defined over $R$. We consider the universal smooth hyperplane section $\mathbb{X}_{R} \rightarrow V_{R}$ of $Y$ over $R$. We denote the universal hyperplane section over $\bar{F}$ (resp. $k$ ) by $\mathbb{X}_{\bar{F}} \rightarrow V_{\bar{F}}$ (resp. $\mathbb{X}_{k} \rightarrow V_{k}$ ). Let $G_{\bar{F}}$ (resp. $G_{k}$ ) be the monodromy group over $\bar{F}$ (resp. $k$ ).

Let $\ell_{k} \subset\left|\mathcal{L}_{k}\right|$ be a Lefschetz pencil of hyperplane sections of $Y$ over $k$. Let $\Gamma_{k}$ be the Galois group of the function field of $\ell_{k}$. Then there is a monodromy action $\Gamma_{k} \rightarrow \mathrm{O}\left(\mathrm{E}_{s}\right)$ where $s \in \ell_{k}$ is a geometric point, $X_{s}$ is the fiber in the Lefschetz pencil over $s$ and $\mathrm{E}_{s}$ is the orthogonal complement of $\mathrm{H}^{2}\left(Y_{s}, \mathbb{Q}_{l}(1)\right)$ in $\mathrm{H}^{2}\left(X_{s}, \mathbb{Q}_{l}(1)\right)$. Let $H_{k}$ be the Zariski closure of the image of $\Gamma_{k}$ in $\mathrm{O}\left(\mathrm{E}_{s}\right)$. If $\ell_{k}$ is a general Lefschetz pencil, then we have $H_{k}=G_{k}$, c.f. [1, Exposé XVIII, (6.1.6) and (6.1.6.1)]. Here (and below in this proof) "general" means that the Lefschetz pencils that do not satisfy the desired property form a proper closed subset of the Grassmanian of lines in $\left|\mathcal{L}_{k}\right|$. We have the same discussion if we replace $k$ by $\bar{F}$. 
We consider a Lefchetz pencil $\ell$ of hyperplane sections of $Y$ over $R$ such that both $\ell_{k}$ and $\ell_{\bar{F}}$ are general. Since $p \neq 2$, the exceptional locus (i.e. the points on $\ell$ over which the fibers are singular) $T \subset \ell$ is etale over $R[1$, Exposé XVII, § 6]. Thus the specialization map $\pi_{1}\left(\ell_{\bar{F}} \backslash T_{\bar{F}}\right) \rightarrow \pi_{1}^{\text {tame }}\left(\ell_{k} \backslash T_{k}\right)$ is surjective [3, Exposé XIII, Corollaire 2.8], where $\pi_{1}^{\text {tame }}$ stands for the tame fundamental group [3, Exposé XIII, § 2.1.3]. The monodromy action of $\ell_{k}$ factors through $\pi_{1}^{\text {tame }}\left(\ell_{k} \backslash T_{k}\right)$, [1, Exposé XVIII, Corollaire 6.1.10]. It follows that $H_{k}=H_{\bar{F}}$. Therefore $G_{k}=G_{\bar{F}}$ and they are infinite by Lemma 3.2. Then $\mathrm{E}^{\text {alg }}=0$ by Lemma 3.1.

We now prove that $\mathrm{NS}\left(Y_{\bar{\eta}}\right) \otimes \mathbb{Q} \rightarrow \mathrm{NS}\left(X_{\bar{\eta}}\right) \otimes \mathbb{Q}$ is an isomorphism. By the Weak Lefchetz Theorem, the map $\mathrm{NS}\left(Y_{\bar{\eta}}\right) \otimes \mathbb{Q} \rightarrow \mathrm{NS}\left(X_{\bar{\eta}}\right) \otimes \mathbb{Q}$ is injective. The surjectivity of this map follows from the orthogonal decomposition $\mathrm{H}^{2}\left(X_{\bar{\eta}}, \mathbb{Q}_{l}(1)\right)=\mathrm{H}^{2}\left(Y_{\bar{\eta}}, \mathbb{Q}_{l}(1)\right) \oplus \mathrm{E}$ together with the fact that $\mathrm{E}^{\text {alg }}=0$ and that the cohomology classes in $\mathrm{H}^{2}\left(Y_{\bar{\eta}}, \mathbb{Q}_{l}(1)\right)$ are all algebraic.

Step 2: Proof of Proposition 2.2.

Lemma 3.4. 1) The restriction map $\mathrm{H}^{2}\left(Y_{\bar{\eta}}, \mathbb{Z}_{l}(1)\right) \rightarrow \mathrm{H}^{2}\left(X_{\bar{\eta}}, \mathbb{Z}_{l}(1)\right)$ is injective and the cokernel is torsion free.

2) The Neron-Severi group $\operatorname{NS}\left(X_{\bar{\eta}}\right)$ is torsion free.

3) If char $k=0$, then $\operatorname{NS}\left(Y_{\bar{\eta}}\right) \rightarrow \operatorname{NS}\left(X_{\bar{\eta}}\right)$ is an isomorphism. If char $k=$ $p>0$, then the cokernel of $\mathrm{NS}\left(Y_{\bar{\eta}}\right) \rightarrow \mathrm{NS}\left(X_{\bar{\eta}}\right)$ is killed by some power of $p$.

Proof. Since $X_{\bar{\eta}}$ is a hyperplane section of $Y_{\bar{\eta}}$, we see that

$$
\mathrm{H}_{X_{\bar{\eta}}}^{4}\left(Y_{\bar{\eta}}, \mathbb{Q}_{l} / \mathbb{Z}_{l}\right) \rightarrow \mathrm{H}^{4}\left(Y_{\bar{\eta}}, \mathbb{Q}_{l} / \mathbb{Z}_{l}\right)
$$

is surjective $\left[2\right.$, Exposé XIV], where $\mathrm{H}_{X_{\bar{\eta}}}^{4}\left(Y_{\bar{\eta}}, \mathbb{Q}_{l} / \mathbb{Z}_{l}\right)$ stands for the relative cohomology. Then (1) follows from the Poincaré duality [2, Exposé XVIII].

It follows from [1, Exposé XI, Proposition 1.3] that $\mathrm{H}^{1}\left(X_{\bar{\eta}}, \mathcal{O}\right)=$ $\mathrm{H}^{0}\left(X_{\bar{\eta}}, \Omega_{X_{\bar{\eta}}}^{1}\right)=0$. This can also be computed directly without much difficulty. Thus the group $\mathrm{NS}\left(X_{\bar{\eta}}\right)$ has no $p$-torsion by [1, Exposé XI, Lemma 1.9 (2)]. By [1, Exposé XI, Lemma $1.9(1)]$, if $l \neq p$, the $l$-torsion of $\operatorname{NS}\left(X_{\bar{\eta}}\right)$ is identified with the $l$-torsion of $\mathrm{H}^{2}\left(X_{\bar{\eta}}, \mathbb{Z}_{l}(1)\right)$. Therefore $\operatorname{NS}\left(X_{\bar{\eta}}\right)$ has no prime-to- $p$-torsion by (1).

Statement (3) follows from (1) and Step 1.

Proof of Proposition 2.2. Let $\mathrm{NS}\left(X_{\bar{\eta}}\right)_{00}$ be the sublattice of $\mathrm{NS}\left(X_{\bar{\eta}}\right)_{0}$ generated by $F$ and $\Xi_{X_{\bar{\eta}}}$. We now prove $\operatorname{NS}\left(X_{\bar{\eta}}\right)_{00}=\operatorname{NS}\left(X_{\bar{\eta}}\right)_{0}$. If $p=0$, this 
follows from Lemma 3.4. We assume now that $p \geq 5$. Since the index of $\operatorname{NS}\left(X_{\bar{\eta}}\right)_{00}$ in $\operatorname{NS}\left(X_{\bar{\eta}}\right)_{0}$ is a power of $p$, we only have to prove that if for some integers $a$ and $b$, the element $a F+b \Xi_{\bar{\eta}}$ is divisible by $p$ in $\operatorname{NS}\left(X_{\bar{\eta}}\right)_{0}$, then both $a$ and $b$ are divisible by $p$.

The self-intersection of $a F+b \Xi_{\bar{\eta}}$ on $X_{\bar{\eta}}$ is $-2 b^{2} d$. If $a F+b \Xi_{\bar{\eta}}$ is divisible by $p$ in $\operatorname{NS}\left(X_{\bar{\eta}}\right)_{0}$, then $p^{2}$ divides $-2 b^{2} d$. By assumption, $p$ and $2 d$ are coprime. Therefore $p$ divides $b$. This implies that $a F$ is divisible by $p$ in $\operatorname{NS}\left(X_{\bar{\eta}}\right)_{0}$. The intersection number of $F$ and $\left.\mathcal{O}(0,1,0)\right|_{X_{\bar{\eta}}}$ on $X_{\bar{\eta}}$ is 3. Since $p \geq 5$, we see that $F$ is not divisible by $p$ in $\operatorname{NS}\left(X_{\bar{\eta}}\right)_{0}$. Therefore $p$ divides a. Proposition 2.2 is then proved.

\section{Acknowledgement}

I thank my advisor Shouwu Zhang for his generous guidance and support. I am also grateful to Johan de Jong, Yifeng Liu, Mingmin Shen, Tong Zhang and Xinyi Yuan for many helpful conversations.

\section{References}

[1] Groupes de monodromie en géométrie algébrique. II, Lecture Notes in Mathematics, Vol. 340, Springer-Verlag, Berlin-New York (1973). Séminaire de Géométrie Algébrique du Bois-Marie 1967-1969 (SGA 7 II), Dirigé par P. Deligne et N. Katz.

[2] Théorie des topos et cohomologie étale des schémas. Tome 3, Lecture Notes in Mathematics, Vol. 305, Springer-Verlag, Berlin-New York (1973). Séminaire de Géométrie Algébrique du Bois-Marie 1963-1964 ( $S G A$ 4), Dirigé par M. Artin, A. Grothendieck et J. L. Verdier. Avec la collaboration de P. Deligne et B. Saint-Donat.

[3] Revêtements étales et groupe fondamental (SGA 1), Documents Mathématiques (Paris) [Mathematical Documents (Paris)], 3, Société Mathématique de France, Paris (2003), ISBN 2-85629-141-4. Séminaire de géométrie algébrique du Bois Marie 1960-61. [Algebraic Geometry Seminar of Bois Marie 1960-61], Directed by A. Grothendieck, With two papers by M. Raynaud, Updated and annotated reprint of the 1971 original [Lecture Notes in Math., 224, Springer, Berlin; MR0354651 (50 \#7129)]. 
[4] S. Bosch, W. Lütkebohmert and M. Raynaud, Néron models, Vol. 21 of Ergebnisse der Mathematik und ihrer Grenzgebiete (3) [Results in Mathematics and Related Areas (3)], Springer-Verlag, Berlin (1990), ISBN 3-540-50587-3.

[5] P. Deligne, La conjecture de Weil. I. Inst. Hautes Études Sci. Publ. Math., (1974), no. 43, 273-307.

[6] R. Hartshorne, Algebraic geometry. Springer-Verlag, New YorkHeidelberg (1977), ISBN 0-387-90244-9. Graduate Texts in Mathematics, No. 52.

Department of Mathematics, Columbia University

2990 Broadway, New York, NY 10027, USA

E-mail address: xuehang@math.columbia.edu

Received August 3, 2014 
